\title{
Development and Pilot Testing of the Snackability Smartphone Application to Identify Healthy and Unhealthy Snacks
}

\author{
Lukkamol Prapkree ${ }^{1}$, Masoud Sadjadi ${ }^{2}$, Fatma Huffman ${ }^{1}$, Cristina Palacios ${ }^{1}$ \\ 'Department of Dietetics and Nutrition, Robert Stempel College of Public Health \& Social Work, Florida International University, Miami, FL, USA \\ ${ }^{2}$ Vertically-Integrated Projects, School of Computing and Information Sciences, Florida International University, Miami, FL, USA
}

Objectives: Snacks contribute to the diet quality in youth, which is often poor. Although the US Department of Agriculture (USDA) has guidelines for healthy snacks, this is lost in translation when youth are choosing snacks. To develop a userfriendly app to help identify healthy snacks based on the US Department of Agriculture guidelines and evaluate the app's feasibility, usability, satisfaction, and acceptability. Methods: The 'Snackability' app was developed following the ADDIE (analysis, design, development, implementation, and evaluation) model. The app was pilot tested for 2 weeks among college-age students (18-24 years) using questionnaires and focus groups. Based on the feedback received, the app was improved, and pilottested again. Results: The app had a simple score ( -1 to 11$)$ and feedback (the higher the score, the healthier the snack is). The 1st pilot test among 12 students showed that the app's feasibility and usability were $>50 \%(p<0.05)$. Participants reported that the app was a good way to help individuals select and consume healthy snacks and suggested improving the search process and including average score reports, a 'consumed history' tab, gamification, notifications, and the option to add snacks not found. These were incorporated into the 2 nd version of the app, which was pilot tested among 8 students. Feasibility, usability, and acceptability of the 2nd version were $>50 \%(p<0.05)$. Additional suggestions were to include images for serving size, snack alternatives, and barcode scanning, which were incorporated into the 3rd version. Conclusions: Snackability app was feasible, usable, satisfactory, and acceptable, and several features were improved as suggested by participants.

Keywords: Snacks, United States Department of Agriculture, Youth, Smartphone Application, Behavior

Submitted: May 6, 2019

Revised: July 16, 2019

Accepted: July 16, 2019

\section{Corresponding Author}

Cristina Palacios

Department of Dietetics and Nutrition, Robert Stempel College of Public Health \& Social Work, Florida International University, 1200 SW 8th Street AHC5, Miami, FL 33199, USA. Tel: +1-305-348-3235, E-mail: cristina.palacios@fiu.edu (https://orcid.org/0000-00019437-0376)

This is an Open Access article distributed under the terms of the Creative Commons Attribution Non-Commercial License (http://creativecommons.org/licenses/by$\mathrm{nc} / 4.0 /$ ) which permits unrestricted non-commercial use, distribution, and reproduction in any medium, provided the original work is properly cited.

(c) 2019 The Korean Society of Medical Informatics

\section{Introduction}

Snacking is an important part of the diet among American children, adolescents, and adults [1-4]. Data from the National Health and Nutrition Examination Survey (NHANES) 2015-2016 in individuals aged 20 years and over showed that $94 \%$ consumed snacks on a daily basis, contributing $22 \%$ of their total energy intake, $36 \%$ of their total sugar intake, and $19 \%$ of their total fat and saturated fat intake at each snack occasion [5]. Among college students, we also showed that $98 \%$ consumed snacks daily, with a frequency of 3.9 times per day [2].

Snacks contribute to the diet quality in youth [6], which is 
often poor [7]. Energy-dense and nutrient-poor snacks, including ready-to-eat, highly processed snacks should be considered as unhealthy; however, these are precisely the snacks available in vending machines and consumed more often [8] and widely marketed to youth [6]. Consumption of such snacks can contribute to the increased risk of obesity and cardiovascular diseases $[3,4]$. On the other hand, snacks are also important contributors to key nutrients ( $>20 \%)$, such as vitamins $\mathrm{C}$ and $\mathrm{E}$, magnesium, calcium, potassium, and fiber [1]. Therefore, interventions to identify healthy snacking among youth to promote healthful snacking behaviors are needed [9].

Currently, the US Department of Agriculture (USDA) publishes guidelines for healthy snacks [10]. According to these guidelines, for a snack to be healthy, it must have as the first ingredient a whole grain, a fruit, a vegetable, a dairy product, or a protein food [10]. Also, it must meet the nutrient standards for calories, sodium, sugar, and fats [10]. However, the recommendations from these guidelines are often lost in translation when youth are faced with choosing a snack. There is a need for a practical method to help individuals identify healthy snacks. This could be done using a mobile application (app). This type of technology could be very appealing to youth, as $75 \%$ of adolescents [11] and $92 \%$ of 18 to 29 year olds [12] own a smartphone. Also, about $77 \%$ of smartphone users download apps [13], and 7.6 apps are used on a daily basis [14]. Most of these apps are health-related apps (59\%), such as fitness and nutrition apps [15]. These apps have been reported to lead to behavioral change, such as setting goals, monitoring food intake [16], and consuming fewer calories [17].

The USDA guidelines for snacks aims to help individuals make healthier choices of snacks, especially among youth who have high snack consumption and are also high users of smartphone technology. The translation of these guidelines at the moment of choosing a snack could be done by using an app. Such an app can help individuals make healthy choices of snacks, manage snack intake in moderation, and promote healthful snacking behavior.

Therefore, the aim of this study was to develop a userfriendly app to help identify healthy snacks based on the USDA guidelines. We also evaluated the app's feasibility, usability, satisfaction, and acceptability, and explored experiences and feedbacks with the app among college students. This app could be very relevant in healthcare as it could be recommended to individuals to help them choose healthy snacks, which could help improve their diet quality. This, in turn, can help prevent or reduce obesity and most chronic conditions.

\section{Methods}

The 'Snackability' app was designed to help identify healthy and unhealthy snacks using a scoring system based on the USDA guidelines for healthy snacks. It was developed following the instructional design model of 'Analysis, Design, Development, Implementation, and Evaluation (ADDIE Model)' [18]:

(1) Analysis phase: A thorough review of the available smartphone apps relevant to snacks was conducted to identify similar apps. For the apps providing a snack score, the scoring systems of these apps were also reviewed. Our search did not identify other mobile apps that translated the USDA guidelines for healthy snacks.

(2) Design phase: The app's goal was to help and guide individuals to make smart and healthy choices of snacks whenever they had snack occasions based on the USDA guidelines for healthy snacks.

(3) Developmental phase: Several tasks were conducted in this phase.

- Obtain a comprehensive snack database: All lists of snack items available in vending machines and shops were obtained at Florida International University (FIU). The database contained information on portion size, calories, percentages of calories from fat and saturated fat, trans fat (g), sodium (mg), and percentage of sugar by weight per portion size of a snack. This local database was stored in MySQL (an open-source relational database management system) workbench. In addition to our snack database, we connected with the USDA Food Composition Database [19] by using the non-deterministic programming (NDP) application programming interface (API) to get representational state transfer (REST) access to this database.

- Review the Smart Snack Guideline from the USDA [10]: based on these guidelines, for a snack to be healthy, it must first meet two principles: (i) be a grain product that contains $50 \%$ or more whole grains by weight (have a whole grain as the first ingredient); or have as the first ingredient a fruit, a vegetable, a dairy product, or a protein food; or be a combination food that contains at least $1 / 4$ cup of fruit and/or vegetable using the ChooseMyPlate. gov website [20] for the lists of the first ingredient of the five food groups; and (ii) meet the nutrient standards for calories ( $\leq 200$ calories), total fat ( $35 \%$ of calories), saturated fat $(<10 \%$ of calories $)$, trans fat $(0 \mathrm{~g})$, sodium $(\leq 200$ $\mathrm{mg})$, and sugar ( $\leq 35 \%$ by weight). 
- Design a unique scoring system: We designed a unique score ranging from 0 to 10 points taking into account the first ingredient, the nutrient standard by portion size, and the processing of foods to deal with the USDA exemptions [10]. Details about this unique scoring system are presented in Table 1. We defined highly processed foods as foods that have been heavily modified from their original form, with the addition of salt, sugar, fat, and/or food additives (substances added to food to preserve flavor or enhance its taste, appearance, or other qualities). One point was subtracted for highly processed foods, and one point was added for foods that were not highly processed. Therefore, the final score ranged from -1 to 11 points. The higher the score, the more compliant it is to the guidelines; therefore, the healthier the snack.

- Design the interface of the app: Users input the name of a snack, portion size, unit, and whether the snack is highly processed. Once the user clicks 'Search', they choose the one that is the most similar to their snack from the list shown. The score is then automatically calculated. The app uses our database first; if the snack is not in our database, then it connects with the USDA database to extract the information from there. Once the score is calculated, the app provides feedback based on the total snack score. It also provides feedback on how to improve the snack. These messages vary depending on the total score of the app and the individual scores. For example, if a snack scored 0 to 5 points, the message displayed would be "This is not a healthy snack, choose another snack!" whereas if the snack scored 10 to 11 points, the message displayed could be "PERFECT score! This snack is very healthy!"

- System configuration and features (Figure 1): The app connects with the USDA Food Composition Database by using NDB API to get REST access to this database and connects with our local database, which is stored in the MySQL workbench. We used React Native (a program to build mobile apps using only JavaScript) to build native applications for both Android and iOS. We also used Redux (a program to write applications that behave consistently and run in different environments) for complex state operations. Firebase (a Google mobile platform for mobile apps) was used for authentication and for user accounts. Web API was built with the NodeJS server (a server that can run JavaScript applications on the server side and the client side built on Google's V8 JavaScript engine) with Express (NodeJS web application framework) to interface with MySQL DB and USDA API. The administrative site runs CRUD (create, read, update, and delete) operations on the local database. Phonetic algorithms were used to improve searches. The Google Cloud

Table 1. Scoring system for the Snackability app

\begin{tabular}{lll}
\hline \multicolumn{1}{c}{ Description } & Score \\
\hline Principle & First ingredient is a fruit, a vegetable, & 2 \\
& a dairy product, or a protein food; or \\
& it is a combination food that contains \\
& at least $1 / 4$ cup of fruit and/or \\
& vegetable
\end{tabular}
Nutrition Calories (kcal) (std: $\leq 200$ calories)
standard

$1.0-50.0 \quad 2$

$50.1-100.0$

$100.1-150.0$

$150.1-200.0 \quad 0.5$

$>200.0 \quad 0$

Total fat (\%) (std: $\leq 35 \%$ of calories)

$0-20.0$

$20.1-35.0 \quad 0.5$

$>35.0$

0

Saturated fat (\%) (std: $<10 \%$ of calories)

$0-4.9$

1

$5.0-9.9$

$\geq 10$

Trans fat (g) (std: $0 \mathrm{~g})$

0

$>0$

Sodium (mg) (std: $\leq 200 \mathrm{mg}$ )

0-140.0 1

$140.1-170.0$

170.1-200.0

$>200.0$

Sugar (\%) (std: $\leq 35 \%$ by weight)

$0-14.9$

15.0-19.9

20.0-24.9

25.0-35.0 0.5

$>35$

Total

Super score

Highly processed food $\quad-1$

Non-highly processed food 


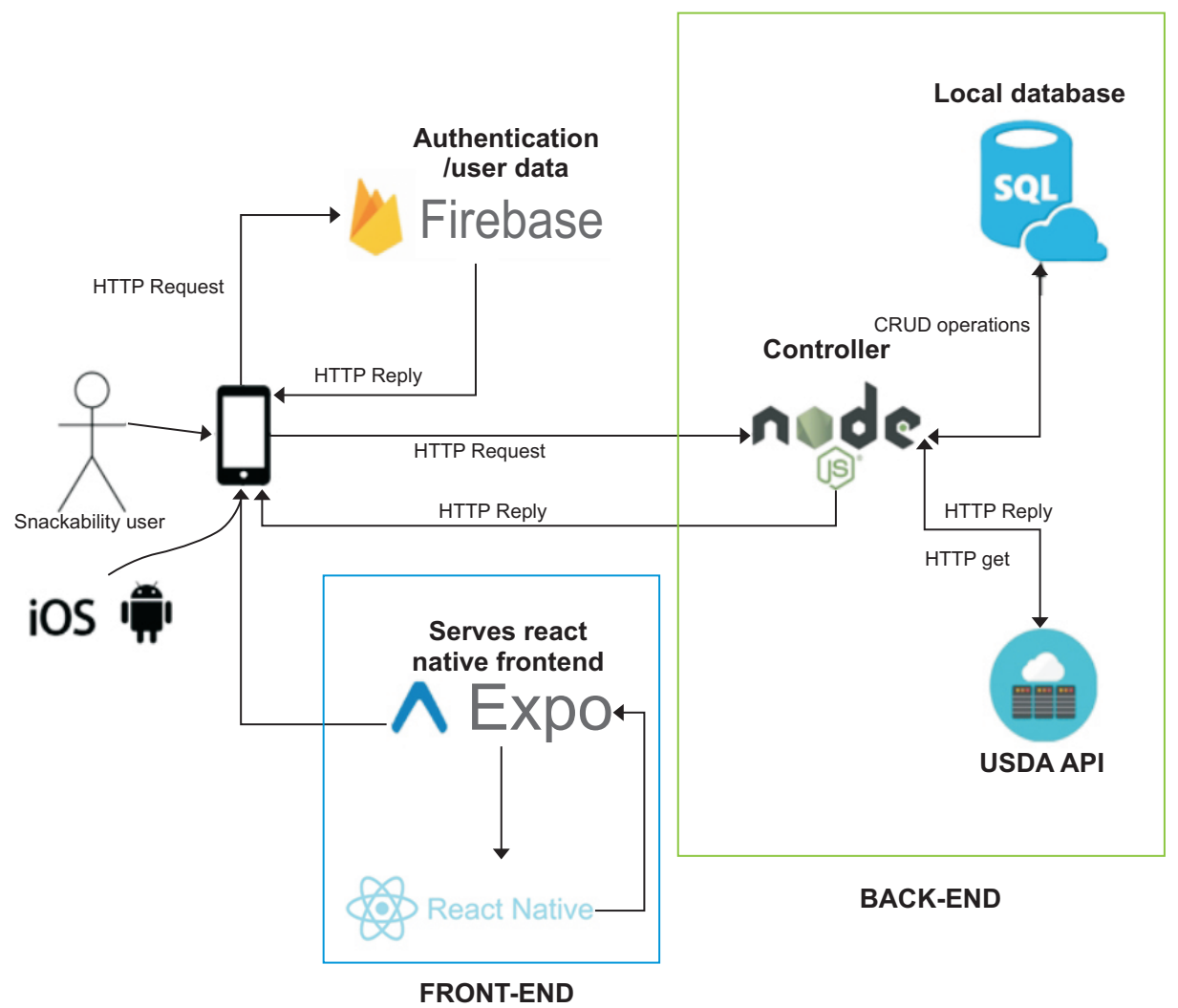

Figure 1. System configuration and features. CRUD: create, read, update, and delete, USDA: US Department of Agriculture, API: application programming interface.
Platform was used for deployment.

(4) Implementation phase: The first version of the app was pilot tested among 12 non-nutrition college students (18-24 years) who owned smartphones at FIU. The participants were asked to complete contact and demographic forms, a short questionnaire about their perceptions of healthy foods and snacks (MB-HSBI-Youth) [21], and their intake of snacks. Then, the participants downloaded the app and were instructed to use it daily for 2 weeks, keeping track of problems they encountered with the app. With input from this first pilot test, improvements were made to the app, and it was pilot tested for a second time among 8 college students (excluding the first group of participants) following the same method. The Institutional Review Board (No. IRB-18-0162AM01) at FIU approved the study, and written consent was obtained from all participants before the initiation of the study.

(5) Evaluation phase: In each pilot test, after the participants used the app for 2 weeks, we evaluated its feasibility, usability, satisfaction, and acceptability with questions using a 5-point Likert scale ranging from strongly agree (5 points) to strongly disagree (1 point). We also asked about the frequency of using the app in the past 2 weeks. The app was considered feasible, usable, satisfactory, and acceptable if $50 \%$ or more of participants answered as 'strongly agreed'/'agreed' or 'yes'. This was compared to those that responded to 'strongly disagreed'/'disagreed' or 'no' using one-sample binomial tests. The binomial test of significance is a type of probability test that is used to examine the distribution of a single dichotomous variable in the case of small samples. Furthermore, the binomial test of significance is non-parametric in nature because it does not involve any parameter. The test was considered statistically significant at $p<0.05$. Statistical analysis was performed using SPSS version 23 (IBM SPSS, New York, NY, USA). The app was also evaluated through focus groups to explore participants' experiences and feedback with the app in the form of audio recordings, which were supported by written notes. This allowed the participants to discuss their experiences with the app and to comment on how to improve it. The focus group discussions were transcribed verbatim after the interview, and the transcripts were analyzed using inductive thematic analysis [22]. Transcriptions were analyzed for quotes and coded using the qualitative software program NVivo12 Analysis Software (QSR International Pty Ltd., Cardigan, UK). Similar codes that represented a dominant theme were grouped to capture the essence of the focus group discussions and ensure the relationships between the research objectives and the research findings. This was done for both the first and second pilot test. 


\section{Results}

\section{Implementation Phase}

In general, among the 20 students recruited (12 for the first pilot test and 8 for the second pilot test), most were able to identify a healthy snack by reading the nutrition facts and agreed that it is important to eat healthy snacks (73.0\%), to be healthy $(79.0 \%)$, and to prevent chronic conditions (76.7\%). A total of $56 \%$ perceived that unhealthy snacks were more accessible and available on campus than healthy snacks, and they consumed these because they could not get healthy snacks from snack machines $(81.0 \%)$ or because they craved these foods (77.5\%). Most students consumed 2 to 4 snacks per day in a typical day (90\%).

\section{Evaluation Phase}

In the 1st pilot test, we found that the app was feasible $(83.3 \%)$ and usable at least five times in the last 2 weeks $(100 \%)$, which was significantly greater than $50 \%(p<0.05)$. However, acceptability and satisfaction were not significantly greater than 50\% ( $p>0.05)$ (Tables 2, 3). Most reported that they would pay or would consider paying for the app (>66\%), and $41.7 \%$ rated the app with 4 or 5 stars. Participants reported that the app was a good way to help people select and consume healthy snacks because the app was simple and provided a score with feedback for each snack. The features that participants liked the most were the score provided by the app for each snack, which created awareness of their snack intake. The feature that participants liked the least was that they could not see improvements over time

Table 2. Feasibility, acceptability, satisfaction, and usability of the Snackability app

\begin{tabular}{lcc}
\hline \multicolumn{1}{c}{ Question } & 1st pilot test $(\mathbf{n}=\mathbf{1 2})$ & 2nd pilot test $(\mathbf{n}=\mathbf{8})$ \\
\hline Feasibility questions & & $7(87.5)$ \\
The app was easy to use. & $9(75.0)$ & $7(87.5)$ \\
The app was easy to learn. & $11(91.7)$ & $5(62.5)$ \\
It was easy to find the snack I wanted to buy in the app. & $4(33.3)$ & $4(50.0)$ \\
The app was very quick. & $8(66.7)$ & $7(87.5)$ \\
The app provided me all the information I wanted. & $9(75.0)$ & $7(87.5)$ \\
Acceptability questions & & $7(87.5)$ \\
The icon and font used were attractive and recognizable. & $7(58.3)$ & $7(87.5)$ \\
I felt very comfortable using the app. & $9(75.0)$ & $7(87.5)$ \\
I liked the layout of the app. & $4(33.3)$ & $5(62.5)$ \\
I liked the screen of the app. & $5(41.7)$ & $7(87.5)$ \\
Satisfaction questions & & $8(66.7)$ \\
I would use this app again. & $7(58.3)$ & $8(100)$ \\
Overall, I liked the app. & & \\
Usability questions & $12(100)$ & \\
Used the app five or more times during 2 weeks. & & \\
\hline
\end{tabular}

Values are presented as number (\%).

Table 3. Feasibility, acceptability, satisfaction, and usability of the app among participants

\begin{tabular}{|c|c|c|c|c|}
\hline \multirow{2}{*}{ Parameter } & \multicolumn{2}{|c|}{1 st pilot test $(n=12)$} & \multicolumn{2}{|c|}{2 nd pilot test $(n=8)$} \\
\hline & n (\%) & $p$-value & n (\%) & $p$-value \\
\hline Feasibility ( $\geq 50 \%)$ & $10(83.3)$ & $0.023^{\star}$ & $7(87.5)$ & $0.040^{*}$ \\
\hline Acceptability ( $\geq 50 \%)$ & $7(58.3)$ & 0.415 & $7(87.5)$ & $0.040^{*}$ \\
\hline Satisfaction $(\geq 50 \%)$ & $8(66.7)$ & 0.214 & $5(62.5)$ & 0.385 \\
\hline Usability ( $\geq 50 \%)$ & $12(100)$ & $0.000^{*}$ & $8(100)$ & $0.005^{*}$ \\
\hline
\end{tabular}

A binomial test was performed to test each hypothesis.

${ }^{*} p<0.05$ considered significant. 


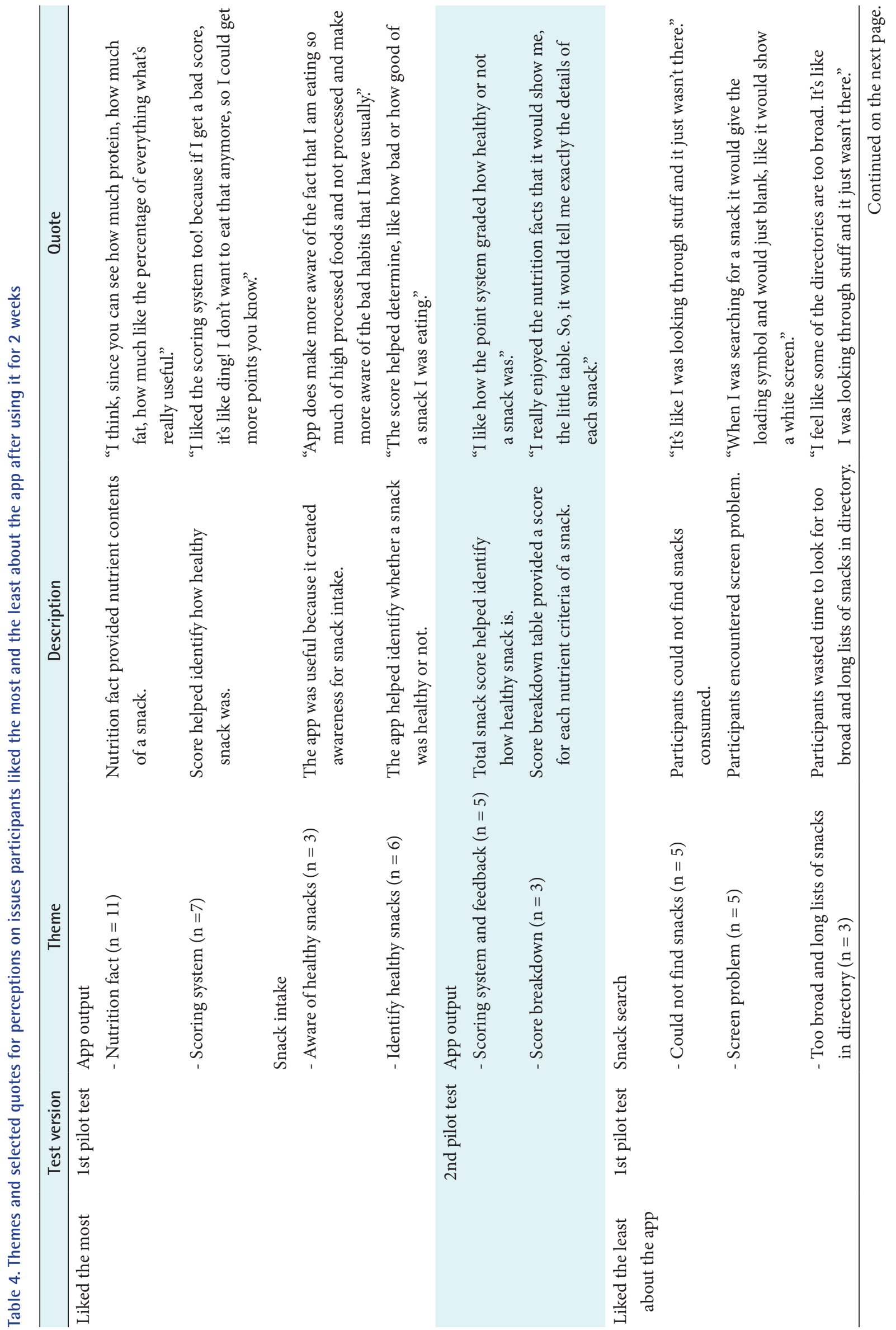




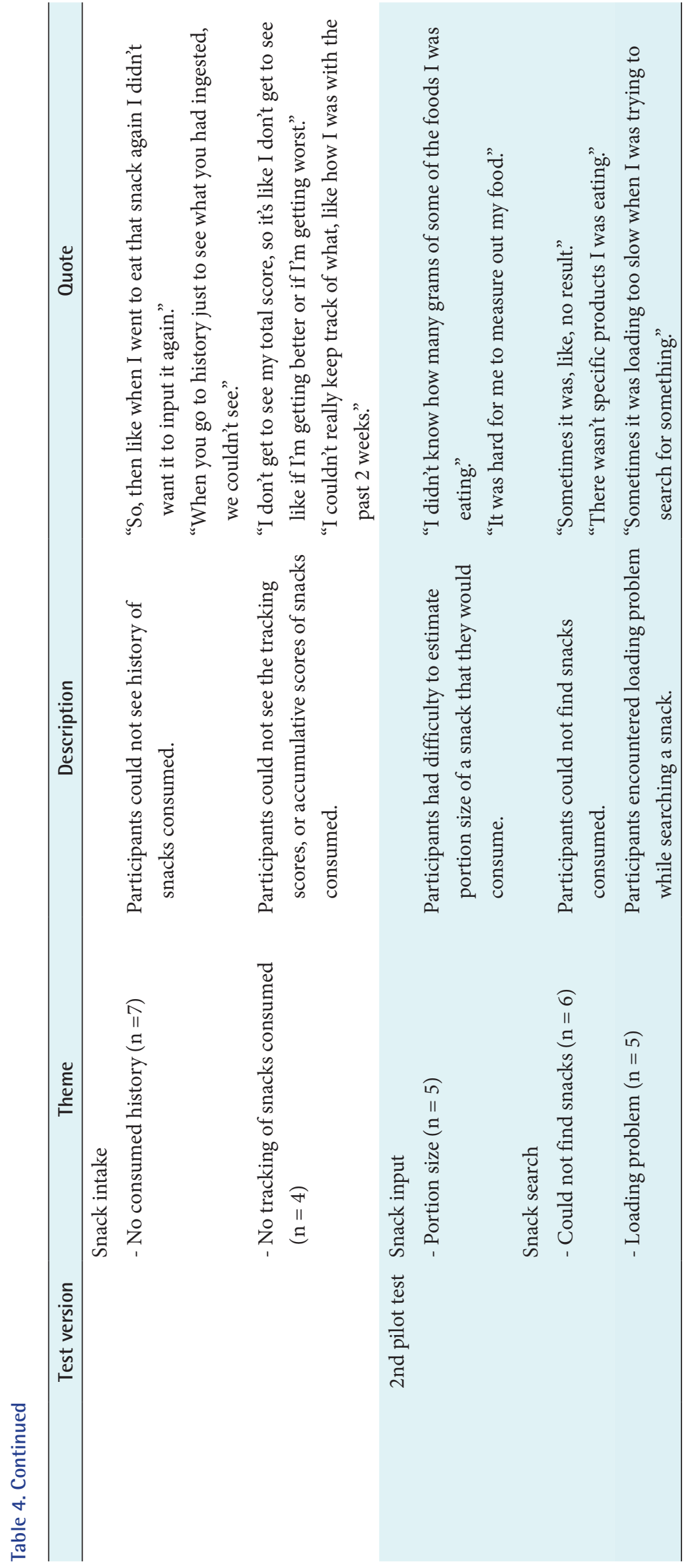




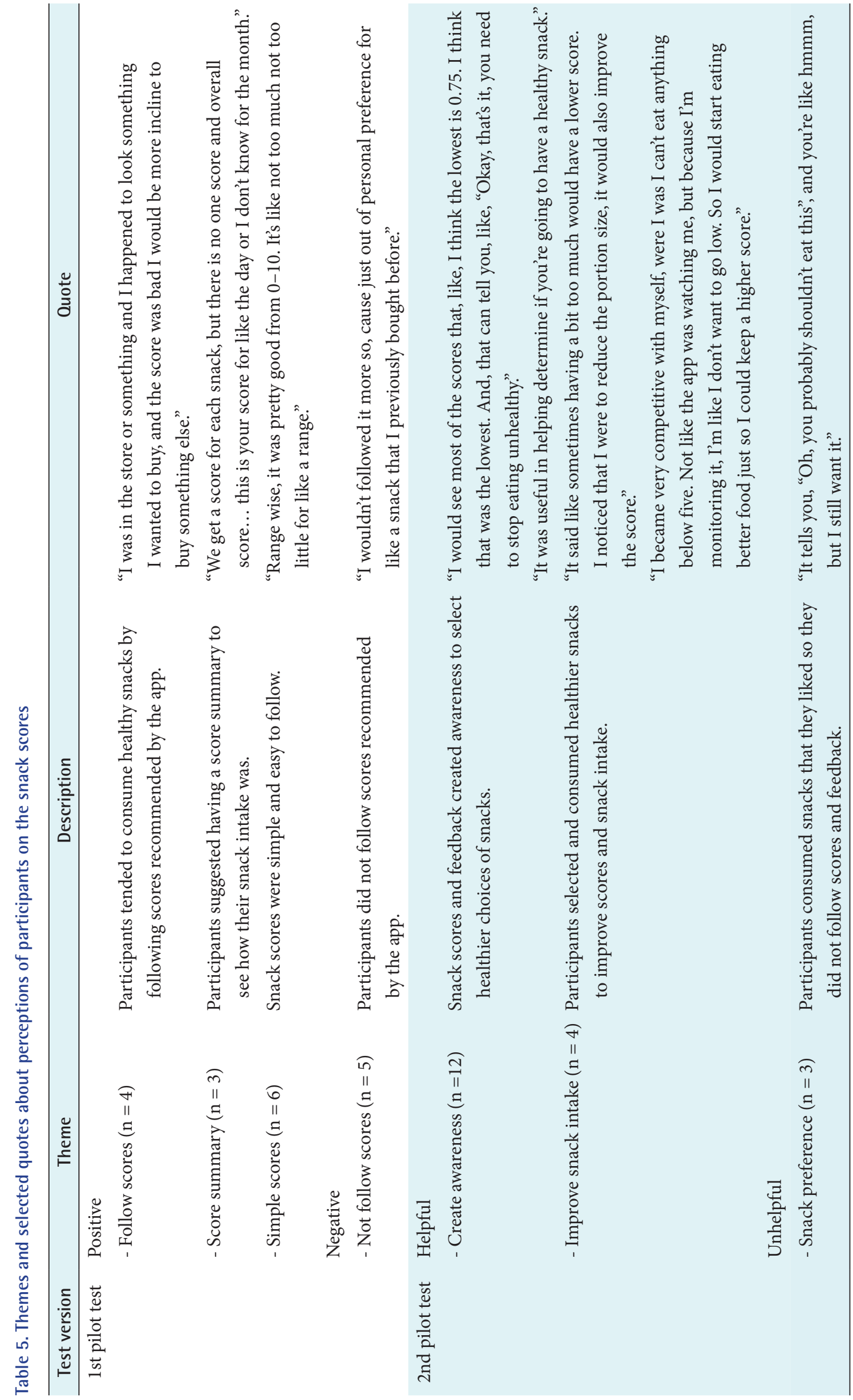


and that some snacks were missing or not easy to find (Table 4). Participants thought that the snack scores were simple and encouraged participants to follow them; however, some participants did not follow the snack scores (Table 5). Participants also reported problems with the app, such as issues with the search page, lack of overall score average, and lack of motivation and reminders to use the app. Accordingly, they suggested improving the snack search feature, including a score breakdown from the total score, including a daily/ weekly average score report or history tab for consumed snacks to keep track of their snack intake, including gamification to motivate them to use the app, including notifications to remind them to use the app, including the ability to add a snack to the app, including snack alternatives if the score is low, and the possibility of barcode scanning. Several of these suggestions were incorporated into the 2 nd version of the app.

In the 2nd pilot test, we found that the app was feasible $(87.5 \%)$, acceptable $(87.5 \%)$, and usable at least five times in the last 2 weeks (100\%), which was significantly greater than $50 \%(p<0.05)$. However, satisfaction $(62.5 \%)$ was not significantly greater than $50 \%(p>0.05)$ (Tables 2,3$)$. Most reported that they would pay or would consider paying for the app (62.5\%), and $62.5 \%$ rated the app with 4 or 5 stars. The features participants liked most were the scoring system, the score breakdown table, and the feedback quotes. The least liked features were the difficulty of estimating portion size and the snack search, as some specific snacks were still missing (Table 4). The participants suggested including images showing different sizes of snacks (small, medium, and large) or a typical serving size of snacks, nutrition information about healthy and unhealthy snacks, alternative healthy snacks, notifications to use the app, and barcode scanning. Most of these suggestions were incorporated into the $3 \mathrm{rd}$ version of the app as shown in Figures 2 and 3.

The 3rd version of the Snackability app has the following three main functions:

(1) Search page: It consists of searching for a snack (scan barcode or type snack name), adding a portion size (based on a portion size guide), selecting whether a snack is highly processed or not, calculating a snack score, reporting total snack scores and breakdown scores (with specific feedback messages about the score), and a consumed button (to register the snack consumed).

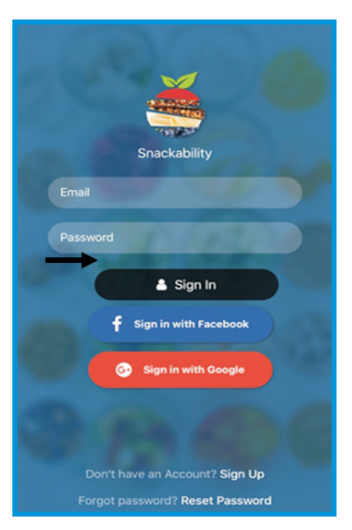

Snackability sign in page

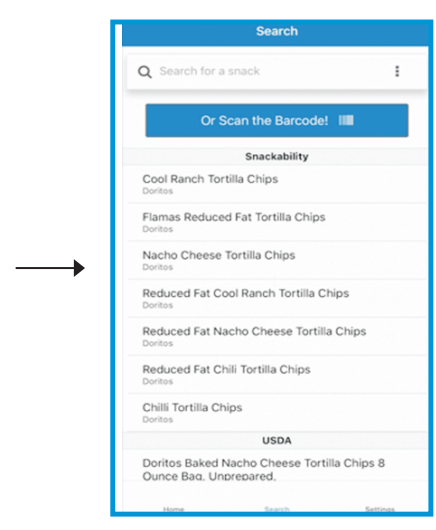

Search for a snack

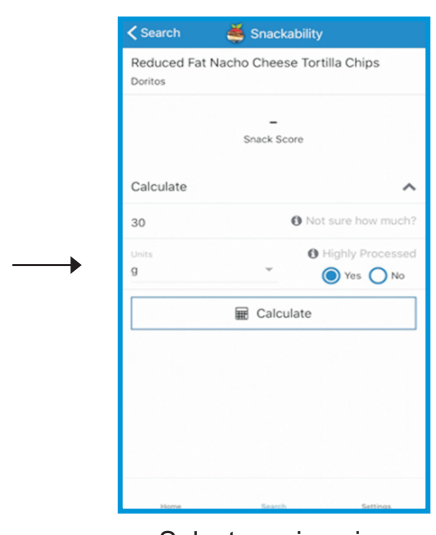

Select serving size

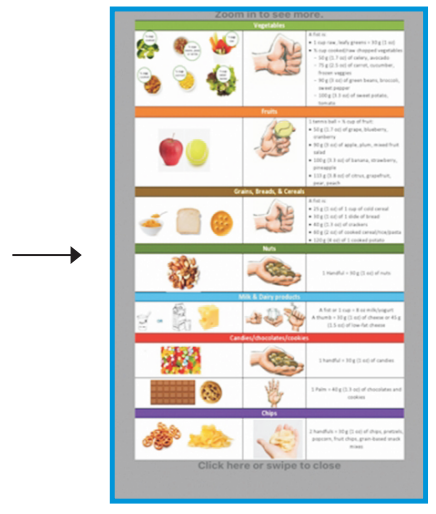

Portion size guide
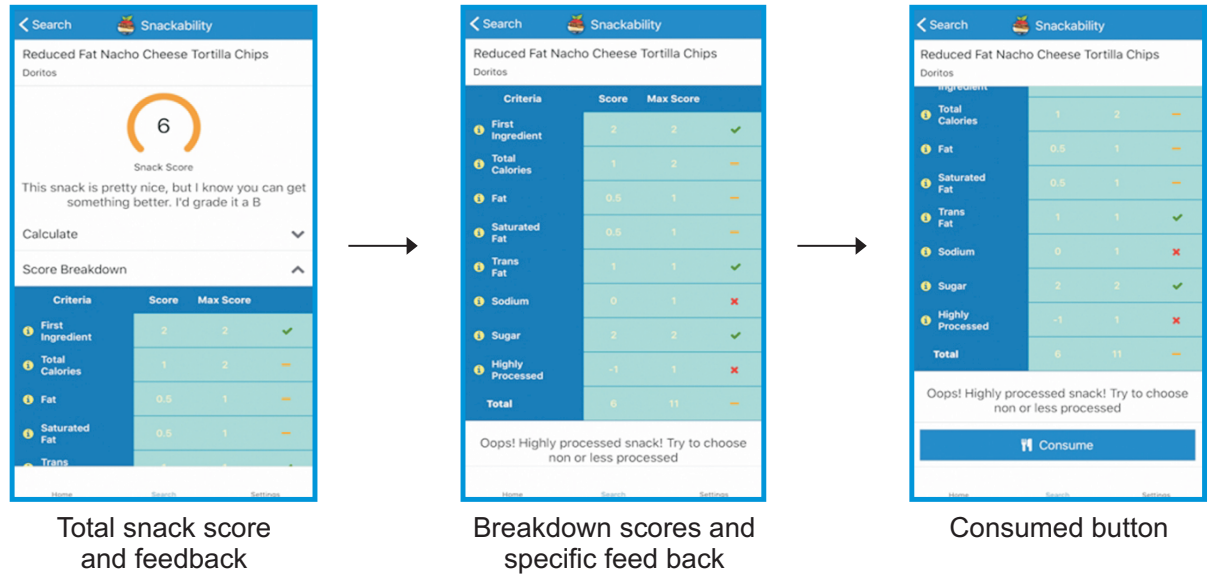

Figure 2. Interface of searching and reporting score of the 3rd version of the Snackability app. 


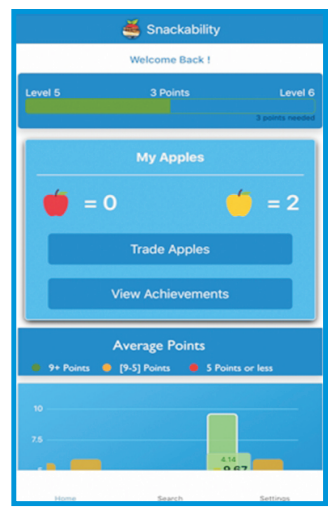

Gamification

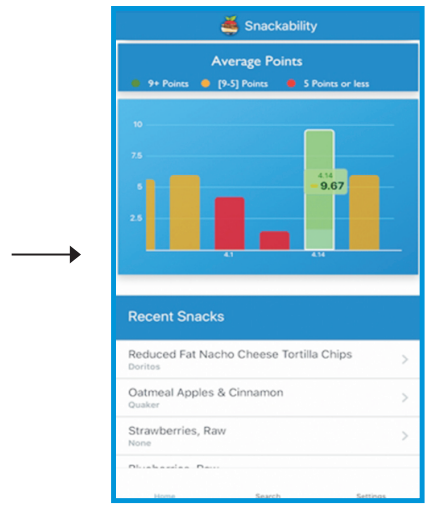

Progress graph

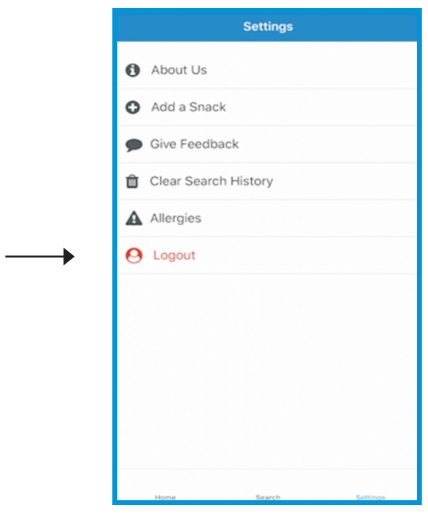

Settings

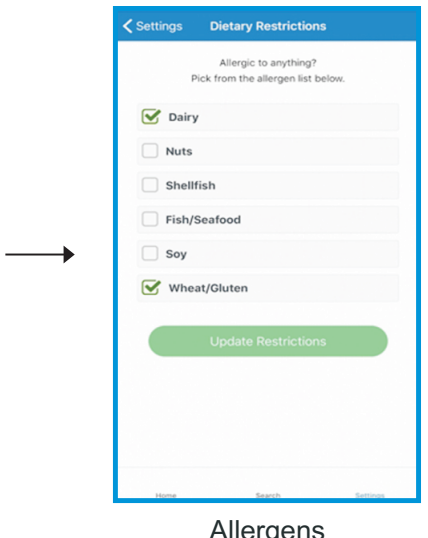

Allergens

Figure 3. Interface of home and settings page of the 3rd version of the Snackability app.

(2) Home page: It consists of the gamification components (level and awards achieved) and reporting components (average daily score shown in a graph and consumed snack history).

(3) Settings page: It consists of adding a snack (users can submit a new snack to the administrators), giving feedback (users can send feedback or comments about the app via email), and allergen restrictions (users can add allergens that they would like the app to report on).

\section{Discussion}

The 'Snackability' app was designed to translate the USDA guidelines on healthy snacks [10]. This tool could be very helpful when youth are faced with the decision of choosing a snack, particularly at the moment of purchase from vending machines. It provides feedback on how to improve the selection of snacks. It also tracks snack consumption and helps motivate individuals to keep using the app with gamification. To the best of our knowledge, this is the first app to have such features. This app could be recommended in the future to help users improve their diets, which could help prevent obesity and other health conditions related to nutrition.

In developing the app, the most challenging step was obtaining the comprehensive snack database from our snack database and USDA database and incorporating them into the app. The first version of the app could retrieve only the USDA database, and the programing language did not match well with the USDA food database. This caused the problems regarding the search feature reported in the first version of the app, in which many participants could not find specific snacks in the app. For the second version of the app, the programing language was improved to match with the USDA database and with our database. This improved the search feature, although there were still some specific brands and products that were not easy to find in the app. For the third version of the app, barcode scanning was incorporated to facilitate snack search.

A comparison between the 1 st and 2 nd pilot test (Table 2) showed that the most items for feasibility, usability, satisfaction, and acceptability improved. However, satisfaction still was not greater than $50 \%$, and this could be related to difficulty with finding some specific snacks and with estimating portion size. Therefore, barcode scanning and a picture guide of portion sizes were incorporated into the $3 \mathrm{rd}$ version of the app.

The pilot testing of the improved app (2nd version) showed that it was feasible, acceptable, and usable by $>50 \%$. Participants reported that the app helped create awareness for selecting healthier snacks, tracking their progress, and motivating them to improve scores and snack intake. The most liked features were the scoring system and feedback quotes, which they reported helped them select healthy snacks. They mentioned that the score breakdown helped them learn which snacks had too many calories, sugars, and fat, which helped in the selection of healthier snacks. Participants also mentioned that there were snacks they thought were healthy but upon obtaining their scores, they learned that they were not healthy; thus, the app helped them select a healthier snack. Furthermore, participants noticed that if they reduced the portion size of their snack, it would also improve the score.

In general, participants reported that the app helped them select, consume, and track consumption of healthy snacks. Therefore, Snackability has the potential to improve snack intake among youth. This is important because studies of snacking trends have shown a significant increase in calorie 
intake from snacks per capita per day since 1977 among American children, adolescents, and adults [3,4]. Energydense and nutrient-poor snacks can contribute to high energy intake and weight gain [8]. Also, Snackability may lead to behavioral change by ways of app engagement, convenience, and ease of use, which can help reduce barriers and increase adherence [16]. Also, Snackability provides feedback and allows self-monitoring of behavior, which are the two most commonly used techniques in behavioral change [23].

Furthermore, the 'gamification' component of the app allowed participants to gain points by the quality of the snacks consumed; these points are used to achieve higher levels (from level 1 to level 100). This could be an important incentive to use the app at each snack occasion and to help self-monitoring of snack intake. This was evidenced by the following comment from a participant "I became very competitive with myself, where I set a goal of not eating anything below five. Not like the app was watching me, but because I'm monitoring it, I'm like I don't want to go low. So I would start eating better food just so I could keep a higher score." Gamification has been used in other apps for behavioral change techniques that are commonly based on feedback, self-monitoring, and goal-setting [23].

This study provides critical insights into the development of mobile apps, which can serve as a framework for future app development to improve healthy eating. Furthermore, pilot testing of the app was essential to obtain feedback from the target population to improve the app to meet their needs, acceptability, and satisfaction. To the best of our knowledge, this is the first study to pilot test different versions of the app based on users' feedback. This study had limitations, such as a small sample size and lack of a random process to recruit participants. However, participants were recruited from various departments on campus to improve the range of responses.

In conclusion, the Snackability app translates the USDA guidelines for healthy snacks into a simple output score to help and guide individuals to make smart and healthy choices of snacks. We showed that it was feasible, usable, satisfactory, and acceptable, and several features were improved as suggested by participants. The app proved to be simple to use, providing a snack score and breakdown, feedback, tracking, and gamification. Therefore, Snackability can be used as a tool for individuals to help identify healthy snacks. In the future, Snackability should be tested to assess its effectiveness for improving the quality of snack intake and preventing obesity and other chronic conditions.

\section{Conflict of Interest}

No potential conflict of interest relevant to this article was reported.

\section{Acknowledgments}

This study was conducted with support in part by internal funds from FIU (No. 8000010077). We thank the students that helped in the development of Snackbility app: Bérénice De La Mota, Frank Hernandez, Bertha Perez, Christian Canizares, Carolina Karthik, Jessiel Benitez, Adriana Serrano, Shafeeque Khan, Jeffery Antoine, Darien Morrison, and Elias Garcia. We also thank the students that helped transcribe the audio recordings: Carolina Velasco and Liah Fernandez.

\section{ORCID}

Lukkamol Prapkree (http://orcid.org/0000-0002-2302-190X) Masoud Sadjadi (http://orcid.org/0000-0003-3915-6603)

Fatma Huffman (http://orcid.org/0000-0001-8513-1648) Cristina Palacios (http://orcid.org/0000-0001-9437-0376)

\section{References}

1. Sebastian RS, Goldman JD, Wilkinson Enns C. Snacking patterns of US adolescents: what we eat in America, NHANES 2005-2006 [Internet]. Washington (DC): Food Surveys Research Group; 2010 [cited at 2019 Jul 1]. Available from: https://www.ars.usda.gov/research/ publications/publication/?seqNo115=255987.

2. Fabian C, Pagan I, Rios JL, Betancourt J, Cruz SY, Gonzalez AM, et al. Dietary patterns and their association with sociodemographic characteristics and perceived academic stress of college students in Puerto Rico. P R Health Sci J 2013;32(1):36-43.

3. Dunford EK, Popkin BM. 37 year snacking trends for US children 1977-2014. Pediatr Obes 2018;13(4):24755.

4. Dunford EK, Popkin BM. Disparities in snacking trends in US adults over a 35 year period from 1977 to 2012. Nutrients 2017;9(8):E809.

5. US Department of Agriculture. What we eat in America, NHANES 2015-2016: snacks (percent of nutrients by gender and age) [Internet]. Washington (DC): US Department of Agriculture; c2019 [cited at 2019 Jul 1]. Available from: https://www.ars.usda.gov/ARSUser- 
Files/80400530/pdf/1516/Table_25_SNK_GEN_15.pdf.

6. Harris JL, Hyary M, Schwartz MB. Effects of offering look-alike products as smart snacks in schools. Child Obes 2016;12(6):432-9.

7. Lipsky LM, Haynie DL, Liu D, Chaurasia A, Gee B, Li $\mathrm{K}$, et al. Trajectories of eating behaviors in a nationally representative cohort of U.S. adolescents during the transition to young adulthood. Int J Behav Nutr Phys Act 2015;12:138.

8. Njike VY, Smith TM, Shuval O, Shuval K, Edshteyn I, Kalantari V, et al. Snack food, satiety, and weight. Adv Nutr 2016;7(5):866-78.

9. Bailey RL, Dodd KW, Goldman JA, Gahche JJ, Dwyer JT, Moshfegh AJ, et al. Estimation of total usual calcium and vitamin D intakes in the United States. J Nutr 2010;140(4):817-22.

10. US Department of Agriculture. A guide to smart snacks in school [Internet]. Washington (DC): US Department of Agriculture; c2019 [cited at 2019 Jul 1]. Available from: https://fns-prod.azureedge.net/sites/default/files/ tn/508_USDASmartSnacks_508_82218.pdf.

11. Lenhart A. Teens, social media \& technology overview 2015 [Internet]. Washington (DC): Pew Research Center; 2015 [cited at 2019 Jul 1]. Available from: https:// www.pewinternet.org/2015/04/09/teens-social-mediatechnology-2015/.

12. Perrin A. 10 facts about smartphones as the iPhone turns 10 [Internet]. Washington (DC): Pew Research Center; 2015 [cited at 2019 Jul 1]. Available from: https://www.pewresearch.org/fact-tank/2017/06/28/10facts-about-smartphones/.

13. Kelley PG, Consolvo S, Cranor LF, Jung J, Sadeh N, Wetherall D. A conundrum of permissions: installing applications on an android smartphone. In: Blyth J, Dietrich S, Camp LJ, editors. International conference on financial cryptography and data security. Heidelberg: Springer; 2012. p. 68-79.

14. Clement J. Average number of apps used vs. number of apps installed by users in the United States in 2016, by device [Internet]. Hamburg, Germany: Statista; 2018 [cited at 2019 Jul 1]. Available from: https://www.statis- ta.com/statistics/681206/us-app-installation-usagedevice/.

15. Krebs P, Duncan DT. Health app use among US mobile phone owners: a national survey. JMIR Mhealth Uhealth 2015;3(4):e101.

16. West JH, Belvedere LM, Andreasen R, Frandsen C, Hall PC, Crookston BT. Controlling your "App"etite: how diet and nutrition-related mobile apps lead to behavior change. JMIR Mhealth Uhealth 2017;5(7):e95.

17. Turner-McGrievy GM, Beets MW, Moore JB, Kaczynski AT, Barr-Anderson DJ, Tate DF. Comparison of traditional versus mobile app self-monitoring of physical activity and dietary intake among overweight adults participating in an mHealth weight loss program. J Am Med Inform Assoc 2013;20(3):513-8.

18. McGriff SJ. Instructional System Design (ISD): using the ADDIE model [Internet]. University Park (PA): Penn State University; 2000 [cited at 2019 Jul 1]. Available from: https://www.lib.purdue.edu/sites/default/ files/directory/butler38/ADDIE.pdf.

19. US Department of Agriculture. USDA Branded Food Products Database [Internet]. Washington (DC): US Department of Agriculture; c2019 [cited at 2019 Jul 1]. Available from: https://www.ars.usda.gov/northeastarea/beltsville-md-bhnrc/beltsville-human-nutritionresearch-center/nutrient-data-laboratory/docs/usdabranded-food-products-database/.

20. US Department of Agriculture. Start simple with myplate [Internet]. Alexandria (VA): ChooseMyPlate.gov; c2019 [cited at 2019 Jul 1]. Available from: https://www. choosemyplate.gov/start-simple-myplate.

21. Tucker CM, Rice KG, Hou W, Kaye LB, Nolan SE, Grandoit DJ, et al. Development of the motivators of and barriers to health-smart behaviors inventory. Psychol Assess 2011;23(2):487-503.

22. Braun V, Clarke V. Using thematic analysis in psychology. Qual Res Psychol 2006;3(2):77-101.

23. Edwards EA, Lumsden J, Rivas C, Steed L, Edwards LA, Thiyagarajan A, et al. Gamification for health promotion: systematic review of behaviour change techniques in smartphone apps. BMJ Open 2016;6(10):e012447. 